\title{
Deformable Registration in Gynecologic Brachytherapy
}

\author{
(1) Öznur ŞENKESEN
}

Department of Radiation Oncology, Acıbadem Altunizade Hospital, Acıbadem Mehmet Ali Aydın University, İstanbul-Turkey

\begin{abstract}
SUMMARY
Advances in image and computer technologies have enabled the use of advanced techniques in the field of radiation oncology. Different imaging methods and/or data obtained by the same imaging method with different dates require registration process to the reference dataset. Deformable registration performs scaling and shearing movements as well as translation and rotation movements. Thus, the differences between the images are minimized, and the accuracy of the applications is increased. In recent years, 3D-image-based brachytherapy has become more common with the aim to register images for adaptive planning. In this step of the planning process, registration algorithms can provide time and convenience by automatically transferring the defined contours to the registered image. This review examines studies on the use of deformable registration methods in gynecological brachytherapy.

Keywords: Brachytherapy total dose; contour propagation; deformable match; gynecological brachytherapy; parameter collection.

Copyright $\odot$ 2019, Turkish Society for Radiation Oncology
\end{abstract}

\section{Introduction}

In modern brachytherapy (BRT) applications, different imaging modalities data are commonly used in treatment planning, tumor monitoring during treatment course, and follow-up after treatment. In imagingbased gynecological BRT, computed tomography (CT) is preferred as the primary imaging method in applicators reconstruction and organ at risk (OAR) delineation, but it is insufficient to determine target volume. Magnetic resonance imaging (MRI) is the gold standard to identify the target and anatomical structures because of superior soft tissue resolution. CT and MRI as well as nuclear medicine imaging techniques such as positron emission tomography (PET) and single-photon emission tomography (SPECT) provide dynamic data on physiological and metabolic processes, glucose metabolism, and DNA synthesis.

The clinical practice of BRT has moved from 2Dfilm-based system with prescription to Point A and normal tissue point dose reporting as the International Commission on Radiation Units and Measurements (ICRU) Report 38 [1] to 3D-based-image-based BRT with CT or MRI.

Image modalities provide more accurate representation of tumor extent and nearby dose limiting structures, though most centers also continue to report doses to ICRU-defined points. While planning with 3D imaging is common, there has been a move to describe dose in three dimensions, rather than by the ICRU point dose specifications.

Registration and fusion of radiological images taken at different times have an important role in adaptive planning and calculation of total doses in BRT as in external radiotherapy (ERT). $[2,3,4,5]$

\section{Rigid and Deformable Image Registration}

In segmentation tumor volumes and/or normal tissues, image registration and fusion are generally used for planning purposes by combining information from different imaging modalities (e.g., MRI, PET, SPECT, 
and CT). Changes in the treatment plan or prescribed dose can be determined with more accurate matching methods, and a more accurate prediction of the given dose can be achieved. In recent years, image registration in 3D-image-guided BRT, adaptive planning $[3,4]$, computer-aided target and organ delineation $[6,7,8]$ and total dose calculation $[5,2]$ has become increasingly common.

In the rigid registration, a rough estimate of the changes in the total dose distribution between interfraction can be made. This registration method allows the image to be registered by the transformation and/ or rotation movement in all directions. No changes are made to the image for differences in whether the organs are full or empty or the applicator is present or not. After rigid registration, the distances between all points in the image are preserved.

In deformable registration, one image is deformed relative to the other. In addition to the transformation and rotation movements in rigid registration, magnification, and flexion movements are matched to the target image. Each voxel in the image is overlapped with the volume in the other image. From the two images, the target image is reference image. The image to be distorted according to the target is the source image. Each voxel in the source image moves three times the number of voxels in the source image set in three dimensions to overlap the target image. Hybrid registration algorithms that take into account similarity and intensity or both of each point in these images have been developed. When the deformation is large or the boundaries between the structures are not clear, the commonly used densitybased deformable image registration (DIR) algorithm is expected to result in a good matching accuracy.[9] The organ-based DIR algorithms increase accuracy by taking into account both the density and organ contour.[10]

Currently, independent of treatment planning system with DIR, software such as MIM maestro (MIM Software Inc., Cleveland, USA), Velocity (Varian Medical Systems, Palo Alto, USA), and Mirada XD (Mirada Medical Ltd, Oxford, UK) are available for clinical use. Deformable registration software offered with treatment planning systems, developed by different companies, are ABAS (Elekta AB, Stockholm, Sweden), Smart Adapt (Varian Medical Systems, Palo Alto, CA, USA), and RayStation (RaySeach Laboratories, Stockholm, Sweden).

These systems include a large number of DIR algorithms with different optimization methods that take into account the different features of the Demons, BSpline, Finite Elements Model, and so on. It should be noted that there are differences between these algorithms, and the use of rigid algorithms will result in fewer errors if the reliability of the deformable algorithm is not assured.

The accuracy of the registration between the two images can be assessed using metrics such as the dice similarity coefficient and the mean distance agreement (DSC). DSC and MDA give the measure of conformity in contour overlap. DSC takes 1 if the target and source image contours are complately matched, and 0 if there is no overlap.

In their study, Reniers B et al. found that the DSC values of OARs were higher than the target volume.[11]

There is no clear consensus on the clinically meaningful DSC value. Kirby et al. found that DIR was evaluated with a pelvis phantom using 11 different DIR algorithms, and the mean DSC value was 0.83 for rectum. [12] The DIR-based total dose calculation is known to be susceptible to DIR error in the anterior rectal wall and posterior bladder wall. Abe et al., between BRT fractions, high-risk clinical target volume (HR-CTV), showed a reasonable DIR accuracy with DSCs as high as 0.8 for the rectum and bladder.[13]

\section{Deformable Registration in Summation ERT and BRT Doses}

The biological effects of radiotherapy depend not only on the given dose, but also on the dose distribution, treatment volume, dose rate, and duration of treatment. The biological equivalent dose (BED) uses a biological damage model to convert all different doses into a comparable form. Equivalent dose 2 Gy (EQD2) is used to describe the dose to give the same biological damage as an external treatment given $2 \mathrm{~Gy}$ in the fraction. EQD2, as $2 \mathrm{~Gy}$ equivalent, allows the calculation of cumulative doses taken from the fractions doses of BRT and external therapy, which are different from 2Gy.

The Groupe Européen de Curiethérapie European Society for Therapeutic Radiology and Oncology (GEC-ESTRO) published volume-based recommendations for dose prescriptions. [14] Recommendations from Gynaecological (GYN) GEC-ESTRO Working Group (II): When calculating the total dose of ERT and BRT, it is assumed that $100 \%$ of the dose prescribed by ERT is taken by the entire organ while calculating the dose volume histogram (DVH) parameters. The BRT doses are calculated by converting the fraction dose to 2 Gy (EQD2) equivalents. GEC-ESTRO suggested the use of DVH parameters with the assumption that hot spots occur in the same anatomical position in each fraction as the worst scenario in collecting fractional BRT doses. [2,15]

According to these recommendations, since a combined dose volume histogram cannot be obtained by calculating the total doses of BRT with EBRT, only the 


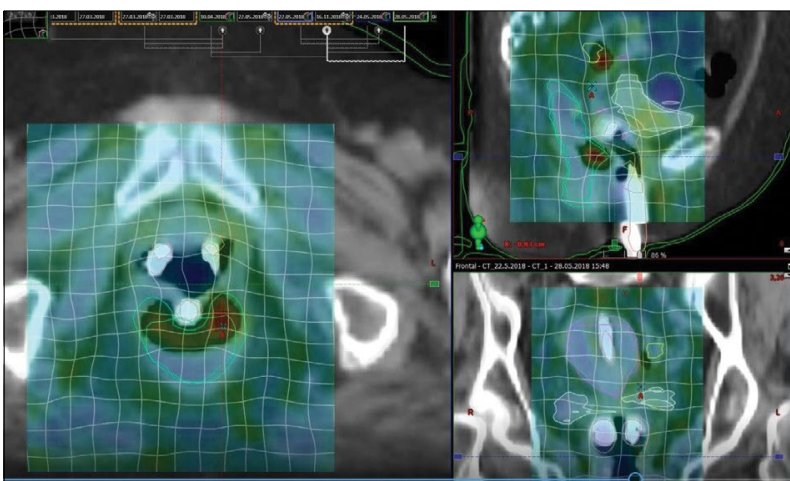

Fig. 1. First BRT fraction image set taken as a reference, DIR with the second BRT fraction image set taken as the source image. It shows the regions where the blue deformation is small and the red deformation is large.

maximum total doses of OARs have been reported using the method of "DVH parameter adding."

However, the DVH parameters can easily change because of organ deformation and movements, or because the applicators are in different place in each fraction. A high estimate of the dose of OAR causes a low dose to be given to the target.

In today's adaptive treatments, it is important for both EBRT and BRT to know the exact dose given to the tumor, as well as normal tissue doses, and to take into account the deficiencies in the previous dose distribution, to plan for subsequent treatments.[2,15]

The use of the 3D total dose distribution rather than the collection of DVH parameters of each fraction dose is preferred as it will increase accuracy. However, it is not very easy to obtain the total dose. The factor that complicates the summation of BRT treatment doses with EBRT is the change in the size of the primary tumor, as well as applicators that significantly deform the pelvic anatomy of normal tissues. Two approaches are used to deal with this uncertainty: (1) register of each data set of EBRT treatment planning sections with rigid methods with BRT sec- tions, or (2) matching of these two different data sets with the deformable method.[2]

BRT dose distributions are characterized by a high dose gradient around the applicator and OARs. This specificity to BRT makes treatment more sensitive to geometric uncertainties associated with organ deformation and source positioning. Because the anterior wall of the rectum and the posterior wall of the bladder are the areas most exposed to radiation, the $\mathrm{D}_{2 \mathrm{~cm} 3}$ dose calculation is the most affected area, especially when the RAO boundary walls are registered incorrectly.

In Figure 1, the first BRT fraction image set taken as reference, when the image set of the second BRT fraction is taken as the source image, is registered with DIR. The magnitude of the deformation is visually distinguishable from the change in grid line and the color scale from blue to red.

Figure 2 shows the deformable registration of the ERT imaging series with the 1.BRT fraction selected as the reference. In addition to the location and size changes of the organs, the presence of the applicator indicates that the registration is not sufficiently successful.

The accuracy of deformable algorithms may not always be sufficient for BRT applications. The presence of the BRT applicator makes this difficult for the algorithms. The organ deformation and intensity change caused by the applicator is the most important factor that reduces DIR accuracy. When the HU (Hounsfield unit) value of the structures in the applicator or applicator scatter changes, the DIR accuracy increases. [2]

Teo et al. investigated whether the use of DIR in collecting doses in the EBRT and BRT plans resulted in differences in the doses of $\mathrm{D}_{2 \mathrm{~cm} 3}$ reported for the rectum and bladder. The DIR software used in the study deforms one CT by matching image density, air pockets within the rectum and bladder, artifacts inside the rectum and bladder, which will not show a significant agreement between different image sets, because inconsistent CT numbers between image pairs will compromise the accuracy of image matching. They were

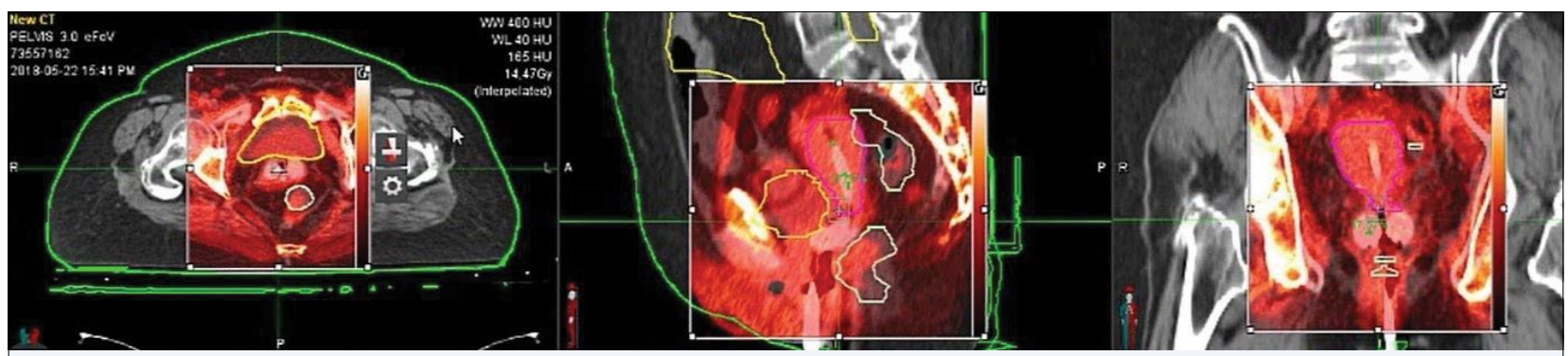

Fig. 2. The deformable registration of the ERT imaging series with the 1.BRT fraction selected as reference. 
used pre-process to correct contrast agents and variations. This pre-process step includes defining the CT numbers of the rectum and bladder contours as 1000 HU, which are similar to dense bones, and thus artificially increase the contrast between the OAR walls and the adjacent soft tissue. In addition, the vaginal packing and applicators used in BRT were contoured to identify the CT numbers as $0 \mathrm{HU}$ (equivalent to water).

The degree of registration of OAR walls between BRT and ERT image series was evaluated by calculating the DSC. The mean and standard deviation of DSC for rectum and bladder were $0.91 \pm 0.03$ and $0.92 \pm 0.03$, respectively. The variation in the positioning of the applicator between BRT fractions and the deformable dose sum because of differences in organ occupancy generally results in lower $\mathrm{D}_{2 \mathrm{~cm} 3}$ values compared to the "parameter adding" that indicates the most irradiated part of each OAR.

In this study, as expected, the total deformable dose of BRT doses was found to be $10.1 \% \pm 9.5 \%$ for the rectum and $7.6 \% \pm 6.5 \%$ for the bladder and $\mathrm{D}_{2 \mathrm{~cm} 3}$ when compared with the parameter adding. These differences were statistically significant for both OARs $(p<0.001)$. In rare cases, very large changes in OAR volumes may result in slightly higher $\mathrm{D}_{2 \mathrm{~cm} 3}$ values. This is because of the less accurate dose distribution between the stretch of RAO walls and image series. These differences in doses, although small, may indicate a correlation between dose volume parameters and late toxicity. The effect of total doses of ERT and BRT on OAR doses is highly dependent on the ERT treatment plan of each patient. In the study, when the EBRT scheme does not include the dose of parametrial, para-aortic, or nodal boost, pelvic dose distributions can be considered as uniform near the rectal and bladder walls closest to the target volume.

In this case, the differences between the calculated doses were not significant with the DVH parameters adding. However, since the OAR dose distribution is largely dependent on the treatment technique and boost rather than on the external boost, it is reported that deformable dose sum may be more appropriate.[2]

In their study, Jamema et al. compared the total sum of the DVH parameters and the dose sum for the bladder and rectum using DIR. When analyzed by two contour-based and density-based DIR algorithms, it is concluded that systematic low estimation can be made when density-based DIR is used, and that direct collection of parameters is a more accurate approach to calculate total dose.[16]

In the study performed by Flower et al., the total DVH parameters $\left(\mathrm{D}_{2 \mathrm{~cm} 3}\right.$ and $\left.\mathrm{D}_{0.1 \mathrm{~cm} 3}\right)$ for the bladder and rectum were compared without DIR and DIR on the planning images of 39 patients with gynecological
BRT. The volumes $\mathrm{VolD}_{2 \mathrm{~cm} 3}$ and $\mathrm{VolD}_{0.1 \mathrm{~cm} 3}$, respectively, consisted of volumes of the $\mathrm{D}_{2 \mathrm{~cm} 3}$ and $\mathrm{D}_{0.1 \mathrm{~cm} 3}$ isodose volumes coincident with the bladder or rectum; the overlap of the volumes was calculated using the DSC.

When the average $\mathrm{D}_{2 \mathrm{~cm} 3}$ and $\mathrm{D}_{0.1 \mathrm{~cm} 3}$ doses were compared, the total doses calculated using DIR were found to be reduced by $2.9 \%$ and $4.2 \%$ for bladder, $5.08 \%$ and $2.8 \%$ for total doses calculated when DIR were not used. To measure the reproducibility of the match, it was shown that $\mathrm{HU}$ was defined by adding $\mathrm{HU}$ to the contours and the reproducibility of $\mathrm{D}_{2 \mathrm{~cm} 3}$ and $\mathrm{D}_{0.1 \mathrm{~cm} 3}$ doses increased. When $\mathrm{VolD}_{2 \mathrm{~cm} 3}$ and $\mathrm{VolD}_{0.1 \mathrm{~cm} 3}$ volumes were re-drawn, the anatomical stability of $\mathrm{VolD}_{0.1 \mathrm{~cm} 3}$ was found to be lower for the rectum and bladder.

Although the dose differences in the study were not statistically significant, the wide range of doses could be clinically significant for some patients. In patients where the total doses of DIR and OAR are smaller than the doses collected without DIR, attention is paid to the possibility that the CTV dose is higher, and vice versa, where these patients are at higher risk of side effects.[15]

Andersen et al. reported a dose difference of 5\% for bladder D0.1 $\mathrm{cm}^{3}$ between simple DVH parameters adding and the total of DIR-based doses in $38 \%$ of patients studied. However, in terms of collecting BRT fraction doses, a small DIR error can lead to considerable dose uncertainty in DVH parameters in high dose gradient region.[17]

Reniers et al. concluded that with a $2 \mathrm{~mm}$ DIR error, the uncertainty in the DVH parameters was approximately $5 \%$ for the bladder and $10 \%$ for the rectum.[11] Therefore, to minimize the uncertainty of DIR-based dose sum, it is necessary to determine the optimal DIR settings.[18] In the studies investigating the methods for evaluating the DVH parameters by calculating the total dose with DIR. $[13,17,19]$ When properly adjusted, the DIR-based dose sum is acceptable to evaluate the total dose distributions of combined radiotherapy for gynecological cancers.

\section{DIR-Based Contour Propagation in Gynecological BRT}

3D volume-based BRT planning requires imaging at different times. The rigid registration of MRI, CT, or PET imaging performed before ERT and the BRT CT or MR image series is used as a standard to determine target and OARs. While the volume-based planning in BRT has advantages, manual contouring and fractionto-fraction plan adaptation require more time. In addition, since applicators remain longer in the patient, the risk of anatomic changes during the fraction and patient discomfort increase. 
New planning strategies were needed to shorten planning time and enhance accuracy. High-risk clinical target volume (HR-CTV) and the availability of DIR registration in contour propagation are investigated. Since DIR can take into account anatomical changes that are not in rigid registration, the ROAs and target volumes can be manually defined in the first fraction; and by removing the contouring step, it can reduce time and increase efficiency.

Chapman $\mathrm{CH}$ et al. investigated the dosimetric effect of the contours created by manually and DIR propagated OAR and HR-CTV. The difference between the D2cc' doses of the bladder, rectum, or sigmoid between the plans of the manual and DIR contours, respectively, was found to be $\mathrm{p}<0.22,0.94$, and 0.40 , the D90 difference for HR-CTV, $p=0.50$, and no statistically significant difference was reported. The use of DIR contours is thought to be feasible because they can be reviewed and manually corrected, if required.[20]

Duane et al., in their study in eight patients, examined the reflection of uncertainties in OAR contours drawn by different individuals in the DVH parameter, respectively, $13.2 \%$ and $9 \%$ of the bladder and rectum $\mathrm{D}_{2 \mathrm{~cm} 3}$ were determined.[21] In a study conducted by Saarnak et al., images of 10 patients were contoured by bladder and rectum volumes by three separate users, and the inter-user variability for $\mathrm{D}_{2 \mathrm{~cm} 3}$ was $10 \%$ and $11 \%$ in the bladder and rectum, respectively.[22] Differences between D2cc and D0.1cc dose sum between DIR and rigid registration may be clinically significant, although the difference in OAR volumes is less than the variability of different individuals. In addition, the uncertainties resulting from the determination of the target volume may affect the DVH parameters of the OAR volumes.[15]

\section{Conclusion}

It may be useful to evaluate the total dose of all fractions instead of collecting DVH parameters in the estimation of HR-CTV and OAR dose in gynecological BRT. The assumption of the worst scenario, the exact overlap of the hot spots in each fraction may not always be accurate, should be considered.

Although studies have aimed at combining ERT and BRT doses, further studies on how to optimize dose collection with DIR in both modalities are needed. Also, DIR algorithms have been shown to provide time and simplicity in this step of the planning process with the option of calculating adaptive plan and total doses as well as automatic contour.

Peer-review: Externally peer-reviewed.
Conflict of Interest: I have no conflict of interest.

Financial Support: I have no financial support.

\section{References}

1. Chassagne D, Dutreix A, Almond P, Burgers JMV, Busch M, Joslin CA. ICRU report 38: Dose and volume specification for reporting intracavitary therapy in gynaecology. Journal of the International Commission on Radiation Units and Measurements 1985;20(1).

2. Teo BK, Bonner Millar LP, Ding X, Lin LL. Assessment of cumulative external beam and intracavitarybrachytherapy organ doses in gynecologic cancers using deformabledose summation. Radiother Oncol 2015;115(2):195-202.

3. Tsuji SY, Hwang A, Weinberg V, Yom SS, Quivey JM, Xia P. Dosimetric evaluation of automatic segmentation for adaptive IMRT for head-and-neck cancer. Int J Radiat Oncol Biol Phys 2010;77(3):707-14.

4. Kaus MR, Brock KK, Pekar V, Dawson LA, Nichol AM, Jaffray DA. Assessment of a model-based deformable image registration approach for radiation therapy planning. Int J Radiat Oncol Biol Phys 2007;68(2):572-80.

5. Zhang G, Huang TC, Feygelman V, Stevens C, Forster $K$. Generation of composite dose and biological effective dose (BED) overmultiple treatment modalities and multistage planning using deformable image registration. Med Dosim 2010;35(2):143-50.

6. Zhang T, Chi Y, Meldolesi E, Yan D. Automatic delineation of on-line head-and-neck computed tomography images: toward on-line adaptive radiotherapy. Int J Radiat Oncol Biol Phys 2007;68(2):522-30.

7. Chao KS, Bhide S, Chen H, Asper J, Bush S, Franklin $\mathrm{G}$, et al. Reduce in variation and improve efficiency of target volume delineation by a computer-assisted system using a deformable image registration approach. Int J Radiat Oncol Biol Phys 2007;68(5):1512-21.

8. Weiss E, Wijesooriya K, Ramakrishnan V, Keall PJ. Comparison of intensity-modulated radiotherapy planning based on manual and automatically generated contours using deformable imageregistration in four-dimensional computed tomography of lung cancerpatients. Int J Radiat Oncol Biol Phys 2008;70(2):572-81.

9. Kadoya N, Miyasaka Y, Yamamoto T, Kuroda Y, Ito K, Chiba $\mathrm{M}$, et al. Evaluation of rectum and bladder dose accumulation from external beam radiotherapy and brachytherapy for cervical cancer using two different deformable image registration techniques. J Radiat Res 2017;58(5):720-8. 
10. Weistrand O, Svensson S. The ANACONDA algorithm for deformable image registration in radiotherapy. Med Phys 2015;42(1):40-53.

11. Reniers B, Janssens G, Orban de Xivry J, Landry G, Verhaegen F. Dose distribution for gynecological brachytherapy with dose accumulation between insertions: Feasibility study. Brachytherapy 2016;15(4):50413.

12. Kirby N, Chuang C, Ueda U, Pouliot J. The need for application-based adaptation of deformable image registration. Med Phys 2013;40(1):011702.

13. Abe T, Tamaki T, Makino S, Ebara T, Hirai R, Miyaura $\mathrm{K}$, et al. Assessing cumulative dose distributions in combined radiotherapy for cervical cancer using deformable image registration with pre-imaging preparations. Radiat Oncol 2014;9:293.

14. Pötter R, Haie-Meder C, Van Limbergen E, Barillot I, De Brabandere M, Dimopoulos J, et al. Recommendations from gynaecological (GYN) GEC ESTRO working group (II): concepts and terms in 3D image-based treatment planning in cervix cancer brachytherapy$3 \mathrm{D}$ dose volume parameters and aspects of 3D image-based anatomy, radiation physics, radiobiology. Radiother Oncol 2006;78(1):67-77.

15. Flower E, Do V, Sykes J, Dempsey C, Holloway L, Summerhayes $\mathrm{K}$, et al. Deformable image registration for cervical cancer brachytherapy dose accumulation: Organ at risk dose-volume histogram parameter reproducibility and anatomic position stability. Brachytherapy $2017 ; 16(2): 387-92$.

16. Jamema SV, Mahantshetty U, Andersen E, Noe KØ, Sørensen TS, Kallehauge JF, et al. Uncertainties of deformable image registration for dose accumu- lation of high-dose regions in bladder and rectum in locally advanced cervical cancer. Brachytherapy 2015;14(6):953-62.

17. Andersen ES, Noe KØ, Sørensen TS, Nielsen SK, Fokdal L, Paludan M, et al. Simple DVH parameter addition as compared to deformable registration for bladder dose accumulation in cervix cancer brachytherapy. Radiother Oncol 2013;107(1):52-7.

18. Kadoya N, Miyasaka Y, Nakajima Y, Kuroda Y, Ito $\mathrm{K}$, Chiba $\mathrm{M}$, et al. Evaluation of deformable image registration between external beam radiotherapy and HDR brachytherapy for cervical cancer with a 3D-printed deformable pelvis phantom. Med Phys 2017;44(4):1445-55.

19. Zhen X, Chen H, Yan H, Zhou L, Mell LK, Yashar CM, et al. A segmentation and point-matching enhanced efficient deformable image registration method for dose accumulation between HDR CT images. Phys Med Biol 2015;60(7):2981-3002.

20. Chapman CH, Polan D, Vineberg K, Jolly S, Maturen $\mathrm{KE}$, Brock KK, et al. Deformable image registrationbased contour propagation yields clinically acceptable plans for MRI-based cervical cancer brachytherapy planning. Brachytherapy 2018;17(2):360-7.

21. Duane FK, Langan B, Gillham C, Walsh L, Rangaswamy $\mathrm{G}$, Lyons $\mathrm{C}$, et al. Impact of delineation uncertainties on dose to organs at risk in CT-guided intracavitary brachytherapy. Brachytherapy 2014;13(2):210-8.

22. Saarnak AE, Boersma $M$, van Bunningen $B N$, Wolterink R, Steggerda MJ. Inter-observer variation in delineation of bladder and rectum contours for brachytherapy of cervical cancer. Radiother Oncol 2000;56(1):37-42. 